\title{
An Extended Cognitive Situation Model for Capturing Subjective Dynamics of Events from Social Media
}

\author{
Yujie Wang \\ La Trobe University \\ wang.y@students.latrobe.edu.au
}

\section{Damminda Alahakoon}

La Trobe University

Daswin De Silva

La Trobe University

\section{Abstract}

The event-indexing situation models are introduced as event models derived from language to facilitate comprehension and memory retrieval. These models explain how fragmental information about events are collected, integrated and updated into a coherent set of views of what the text is about. The models are adopted as the basis of this study as an attempt to capture the event with contextual, dynamic, and social features, as conveyed by the vast volumes of online textual resources. Information in social media is received through highly personalized channels and is reshaped and interpreted in a more individual, segmental and real-time manner. The reprocessed information is then spread at high speed to a wider range of receivers. Therefore, the interpretation of mainstream media content is influenced by ongoing and dynamic contribution of opinions by users empowered by social media. This new phenomenon has not been examined so far from the perspective of the impact on conventional situation models. This paper explores how collaborative and sharing aspects of social media emphasize subjectivity of interpretation of mainstream media and proposes an extended cognitive situation model which better represents event-centric knowledge. This study investigates the mechanisms for constructing and updating the situation models with continuous textual information streamed from heterogeneous forms of media. It also empirically demonstrates how the proposed model can enhance the understanding of subjective aspects of events with dynamic social opinions.

Keywords: social media; natural language processing; text comprehension; collective social opinion

\section{Introduction}

Streams of data are generated from every aspect of our daily life, especially unstructured data narrated in natural language. The streams of textual data often continuously evolve with time, which conveys rich information about events and provide the context of such events, such as temporal-spatial features, individuals involved, objects, causation and goals. The event knowledge embedded in the rapidly increasing volumes of textual data contains considerable potential value for many data-driven decision making applications, and as such has attracted increasing research interests from researchers focusing on natural language processing (NLP), machine learning, event extraction and knowledge representation.

Although existing research has made significant improvements, machine understanding of text remains inadequate considering the massive volume of data to be processed and the depth 
of understanding required. The challenges stem from several aspects, including the complications of natural language, the complex and evolving nature of events, and the seemingly impenetrable interactive and dynamic information exchanging environment enabled by heterogeneous media and diverse platforms. Firstly, knowledge extraction from text largely depends on machine understanding of human language, where most existing research applies either a structural or statistical approach, or a hybrid combination of both. The former stresses on examining the structural representation of text, and the latter focus on the word co-occurrence frequencies and the corresponding statistical distribution patterns. The acquired event knowledge is often static, segmental and isolated from context. Secondly, there is still no consensus among researchers about the definition of an event and how eventbased knowledge should be structured and represented. Focusing on event extraction and tracking, researchers model and represent events either in the form of pre-defined templates or as a list of representative words, terms or concepts depending on their statistical distribution in the texts. Events constructed in these forms are unable to capture the essential situational settings where the events take place. Consequently, it imposes greater challenges for researchers to establish associations between events and the evolutional behaviours of events overtime. In a word, the dynamic and evolving features of real life events are not represented in the knowledge bases built upon these approaches. Lastly, most event extraction research stress on extracting factual knowledge about events from homogenous sources. However, events contain not only objective or factual knowledge such as time, space, individuals, objects, but also subjective information such as causality and intentionality which are subject to interpretation by individuals depending on their emotion, knowledge, understanding and experiences. Traditionally the mainstream media plays an active role in framing the public opinion by viewpoints conveyed in reports. Although the reader can construct an interpretation from such a report, the information flow is one way and do not facilitate a discussion or conversation. With the advent of social media platforms, the public are empowered with exposure to different forms of media such as news, blogs, question and answer (Q\&A) sites, social media platforms. Online users engage in information and opinion creation in a more interactive and influential manner. How such social media data could be captured to augment event knowledge with the public opinion and its evolving dynamics is still an open question for researchers.

Even utilizing current extensive computing power and rapidly growing memory capacity, machine is still unable to achieve human capabilities such as extracting and disambiguating meaning from text, linking and merging fragmental information through situational context, and enhancing the understanding of events with pre-acquired knowledge and experiences. Therefore, to reduce the cognitive gap between machine and human brain, we adopted a couple of psycholinguistic theories focussing on text comprehension, namely the situation models and the event indexing model. The former illustrates the mental representation that human brain deploys to decode text back into the micro-world described by the author (Dijk, Kintsch, \& Dijk, 1983; Johnson-Laird, 1983; R. A. Zwaan, M. C. Langston, \& A. C. Graesser, 1995). The latter emphasises on the construction mechanism of situation models (Radvansky \& Zacks, 2014; R. A. Zwaan et al., 1995).

Situation models and the event indexing model provide means to simulate the process that human brain builds up mental units from narrative texts. As ongoing output of comprehension, the layered and networked mental representation continuously directs reasoning processes and memory related operations (Crowley, Reignier, \& Barranquand, 2009; 
Radvansky \& Zacks, 2014). However, the models are ambiguous in term of guiding empirical study in computing. The theories also lack the explanation of how an information receiver's pre-acquired knowledge impacts the interpretation of the events. Additionally, the models are inapplicable to the interactive information exchanging environment where individual receivers' opinions act as a collective influential force to facilitate the evolution of public opinion overtime.

To address the limitations of event knowledge extraction from heterogeneous media of textual resources, we propose an extended situation model upon the re-evaluation of objective and subjective natures of situational dimensions of events. The revisited model emphasizes capturing events with situational contexts and knowledge enhancement, and elaboration with social opinion dynamics. This study identifies that the subjective features are liable to evolve with influential social opinions, hence we extend the existing model with a social view layer to capture the dynamic evolutions of the collective social opinions overtime.

The rest of this paper is organised as follows: section (2) introduces the related work on natural language processing, event extraction and event knowledge construction with social opinions. Section (3) presents the theoretical basis of this study focusing on the event-indexing situation models. To incorporate the dynamics of social opinions, we revisit the adopted models and propose an extended situation model. Section (4) demonstrates the construction of the extended situation model with a sample dataset to examine the dynamic subjective factors of the constructed situation models. Finally, we discuss the findings and conclude our study in section (5).

\section{Related Work}

Although an event is commonly defined as an occurrence that unfolds a segment of time and specific space (J. Allan, Carbonell, Doddington, \& Yamron, 1998; Chambers \& Jurafsky, 2008; Hogenboom, Frasincar, Kaymak, De Jong, \& Caron, 2016), the content of an event is more than the temporal and spatial aspects. An event is a collection of relations of the involved entities and concepts by essence (Radvansky \& Zacks, 2014). A subset of the situational dimensions are especially important for event construction during text comprehension, including time, space, protagonist and object, causality and intentionality. These interrelated dimensions serve as the skeleton structure of events that supports the cognitive processes, such as relation inference, information analysis and integration, memory retrieval and updating, prediction and decision making (Dijk, 1977; Dijk et al., 1983; W. Kintsch \& Dijk, 1978; Radvansky \& Zacks, 2014; R. A. Zwaan \& G. A. Radvansky, 1998). Noticeably the dimensions of causality and intentionality are often not explicitly phrased in the original texts, and are always inferred and interpreted by the readers depending on their own emotions, knowledge and experiences ( $R$. A. Zwaan \& G. A. Radvansky, 1998), which can be subjective and liable to change with new information.

Therefore, we have investigated the existing work from related research fields including natural language processing, and event extraction. The aim is to find the research gaps in extracting event-based knowledge upon situational context awareness and to enrich the output with collective opinion dynamics in online global conversation environment. 


\subsection{Natural Language Processing (NLP)}

The depth of machine understanding of text largely relies on the natural language processing (NLP) capabilities. NLP is defined as a theory-motivated range of computational technologies for automatic analysis and representation of human language (Cambria \& White, 2014). Existing NLP approaches are adept at conducting lexical and syntactic analysis of text, which examines the structure of sentences and word co-occurrence frequencies (Cambria \& White, 2014). In knowledge extraction and modelling field, retrieving semantics or meanings and performing cognitive 'thinking' based on the extracted facts are still questionable. The challenges come from the complexity of human language.

1. Displacement feature of language. The original sequential order of events is always broken into self-containing chunks and rearranged in accordance to the author's interest.

2. Missing background information. Common-sense knowledge and some situational information are often left out since the information gaps are deemed to be filled up by referring to pre-acquired knowledge or context.

3. Noisiness and redundancy of information. The ambiguous or even controversial information increases the difficulties for machines to resolve coreference, especially in cross-document cases.

Humans comprehend text through a series of much more complex processes taking place almost simultaneously. Human mind can recognize entities mentioned in text and resolve the meanings of word and sentence, identify and merge associated incomplete information according to situational context to understand the events mentioned, and augment the understanding of event with background knowledge and experience to form more comprehensive episodic knowledge (Garnham, 1987; Graesser, Millis, \& Zwaan, 1997). Understanding is a holistic system which integrate different levels of analysing tasks, memory operations to store, retrieve and update extracted knowledge, and mechanisms to integrate local information from text with external knowledge bases. Comparatively, most existing NLP approaches focus more on one or a few aspects of machine understanding of text, than investigating it in a systematic way(Cambria \& White, 2014).

\subsection{Event Extraction Techniques}

Traditionally event extraction refers to the Information Extraction (IE) task, which detects and captures events from unstructured or semi-structured textual sources and generate structured information about the captured events. We studied the event extraction approaches with the focus on event representation and knowledge construction for text understanding. Existing researches fall into two mainstreams: knowledge-driven approach and data-driven approach. Knowledge-driven approaches emphasis on the richness of event knowledge. Event is modelled as a collection of predefined templates in accordance with a set of information extraction rules defined by domain experts (Aguilar et al., 2014; Ahn, 2006; Doddington et al., 2004; Tanev, Piskorski, \& Atkinson, 2008). Data-driven approach stress on the efficiency of information retrieval and event trend identification from large amount of data. Thus researchers conduct statistical analysis of the text structure and classify documents into eventcentric topical categories labelled as lists of representative words with timestamps (James Allan, 2002; J. Allan et al., 1998; Brüggemann, Hermey, Orth, \& Schneider, 2016). A few researches also represent events in a network structure, such as a bank of predicates 
(Kingsbury \& Palmer, 2002), or semantic network of concepts (Baker, Fillmore, \& Lowe, 1998; Gangemi, Hassan, Presutti, \& Reforgiato, 2013). Majority of related work focus on extracting factual relationships between entities on sentence basis, or representation events as statistical calculation results. For example, the template based approaches fill the template slots with the static and fragmental information related to an event, such as person, location, type of event, number of casualties and so on; and the statistical approaches which model events as a list of topical terms can only represent events more ambiguously as words where the meanings of words are not considered (i.e., bank in 'bank account' and 'lake bank' are considered as the same). Therefore, the acquired knowledge of events remains static, segmental and isolated from context.

The above-mentioned limitations further restrain a system to develop sufficient understanding of events in several aspects. Firstly, understanding of event depends on situational context, which is represented as relations of entities or concepts to events. Secondly, event representation should support analysing operations such as distinguishing, comparison and updating of events. Thirdly, various types of associations of events, such as temporal relation, spatial relation, causal relation, are necessary to capture the evolutional dynamics of events. Lastly, understanding of events also depends on external knowledge, such as world knowledge, social knowledge, knowledge of psychological activities and so on (Adolphs, 2009; Langer, 1984; Piaget \& Duckworth, 1970; Van Dijk, 1977).

To sum up, although existing NLP and event extraction and tracking approaches demonstrate strengths in structured and domain-tailored event-related information extraction, or online capture of dynamic topical trend shifting from vast volume of data, these approaches lack two crucial factors to ensure effective text understanding: context and background knowledge. Therefore, comprehension related cognitive processes are not well supported, such as situation reconstruction from text, association establishment of events, recognition of event evolution and knowledge enrichment with external knowledge.

The insufficiency of event knowledge is further reflected in subjective dimensions of events, which largely depend on knowledge, experiences and psychological activities of both individuals and the society. The challenge of modelling subjectivity of event are intensified by the proliferation of social media. Boundaries of conversations have been expanded such that people engage in discussions with broader communities in a more flexible and interactive manner. We propose this new form of information exchange being considered as a form of online global conversation.

\section{Theoretical Basis}

\subsection{Psychological Events Models of Text Understanding}

To improve machine understanding of textual data aiming at bridging the gap between machine and human processing, we examined the cognitive theories related to human comprehension of narrative texts. Prevailing and experimented theories, including Situation Models (Dijk, 1977; W. Kintsch \& Dijk, 1978; R. A. Zwaan et al., 1995) and Event-Indexing Model (Rolf A Zwaan, 1999; R. A. Zwaan et al., 1995) are commonly adopted by researchers of related areas (Crowley et al., 2009; Graesser et al., 1997; Walter Kintsch, 1998; Sanford \& Emmott, 2012) and well support the objectives of this study. The situation models theory abstracts and represents the mental representation human construct during the comprehension processes (Walter Kintsch, 1998; W. Kintsch \& Dijk, 1978; R. A. Zwaan et al., 
1995; R. A. Zwaan \& G. A. Radvansky, 1998). It represents a spatially and temporally bounded event, as fundamental processing units to coordinate the comprehension processes and memory retrieval. The Event-Indexing Model explains the mechanism of situation model construction on sentence basis and how event nodes are activated, updated and associated dynamically via situational dimensions while a reader processes a unit of narrative text (Figure 1).

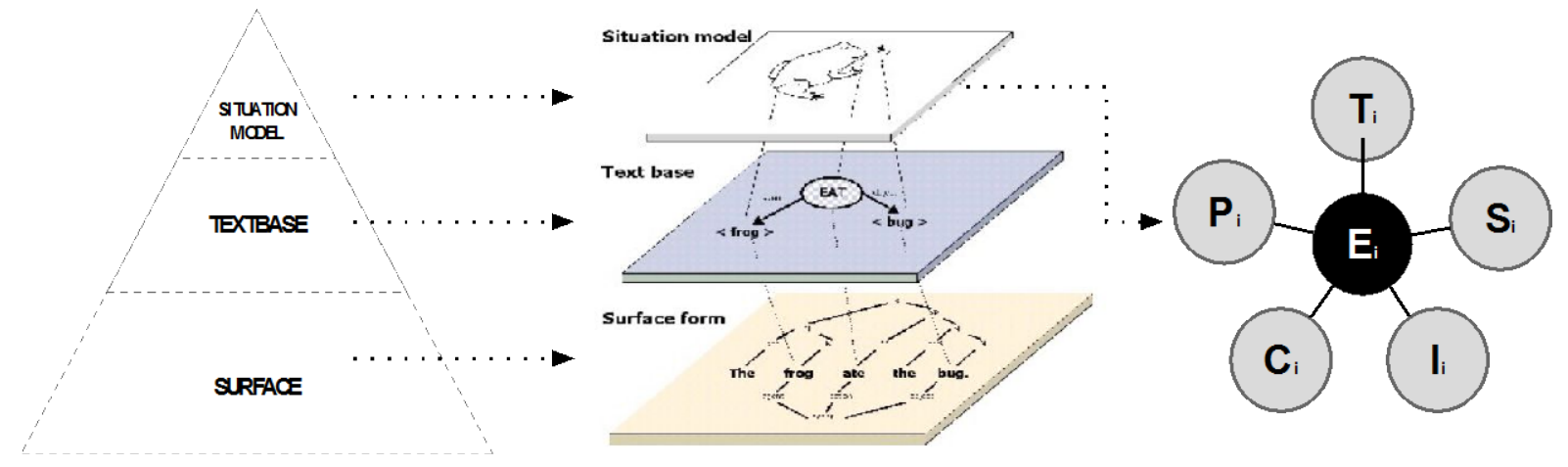

Figure 1: Multi-layer and multi-dimensional mental representation of text comprehension Three layers of multi-dimensional mental representation during human comprehension and the situation model (Dijk et al., 1983; R. A. Zwaan \& Radvansky, 1998)

According these theories, when readers comprehend a story, they construct a multi-level and multi-dimensional mental representation to reflect the micro-world described by the author. The three levels of representations are surface level, propositional level or textbase, and situation model. Surface level represents the environment, which is built upon examining words and syntax of the literal wordings of text (Radvansky \& Zacks, 2014). Identified objects and concepts are networked by extracting propositional relations from sentences, which forms the textbase layer (Walter Kintsch, 1998). The highest level of representation, situation model, represents an event model (Radvansky \& Zacks, 2014). Based on further analysis of the propositional relationship, and enhanced with prior acquired experience/knowledge, a coherent set of situation models are constructed in temporal and causal order as more comprehensive understanding and representation of the plot of the story (Figure 1).

\subsection{Situation Models: Event Based Mental Representation of Text Understanding}

The situation models are introduced as event models derived from language which are represented as components and relations of components. Thus the spatial-temporal framework within which events takes place serves as an essential basis for situation model construction to restore the holistic view of events (Radvansky \& Zacks, 2014; Rolf A Zwaan \& Gabriel A Radvansky, 1998). In event cognition theory, a situation is the condition and status of a collection of events that occur within the same tempo-spatial bound (Radvansky \& Zacks, 2014). A situation model is represented in a network structure with predicates or verbs as the focal points of the events to link the propositions coherently (Rolf A Zwaan, Mark C Langston, \& Arthur C Graesser, 1995; Rolf A Zwaan \& Gabriel A Radvansky, 1998). The five key situational dimensions serve as the supporting indices to assist comprehending, including temporality, spatiality, protagonist and object, and causality and intentionality. The construction of situation models follows a three-layer processes from surface code, textbase to situation model. 
During texts comprehension processes, events serve as the critical focal units in the mental representation which not only connects the events and elements of events but also coordinates the inferencing instructions along situational indices (time, space, protagonist, causality, and intentionality)(Crowley et al., 2009; R. A. Zwaan, 2016; R. A. Zwaan et al., 1995). The situation model theory presents the event-centric mental representation as the fundamental units for knowledge augmentation, inference and retrieval.

\subsection{Event-Indexing Model: Mechanism of Constructing Situation Models}

Event-Indexing Model introduces the mechanisms and processes of how human mind continuously construct and update existing situation models in memory with incoming information (R. A. Zwaan et al., 1995). While a reader comprehends a piece of text, the clauses are parsed into events as the focal units of the situation models. The semantic relations conveyed by the clauses are established through events with the five situational dimensions, time, space protagonist and object, causality and intentionality. The previously constructed situation models are subject to updating with incoming events depending on the continuity of situation settings. Events are updated through a series of situation models. The current model is the model under construction at time $t_{n}$ extracted from clause $c_{n}$. The integrated model of the situations from time $t_{1}$ to $t_{n-1}$ is the global model that is constructed by integrating clauses $\mathrm{c} 1$ to cn-1. Finally, the completed model at $t_{n}$ is the integrated model after the complete set of clauses has been processed (Rolf A Zwaan, 1999; R. A. Zwaan et al., 1995)(Figure 2).

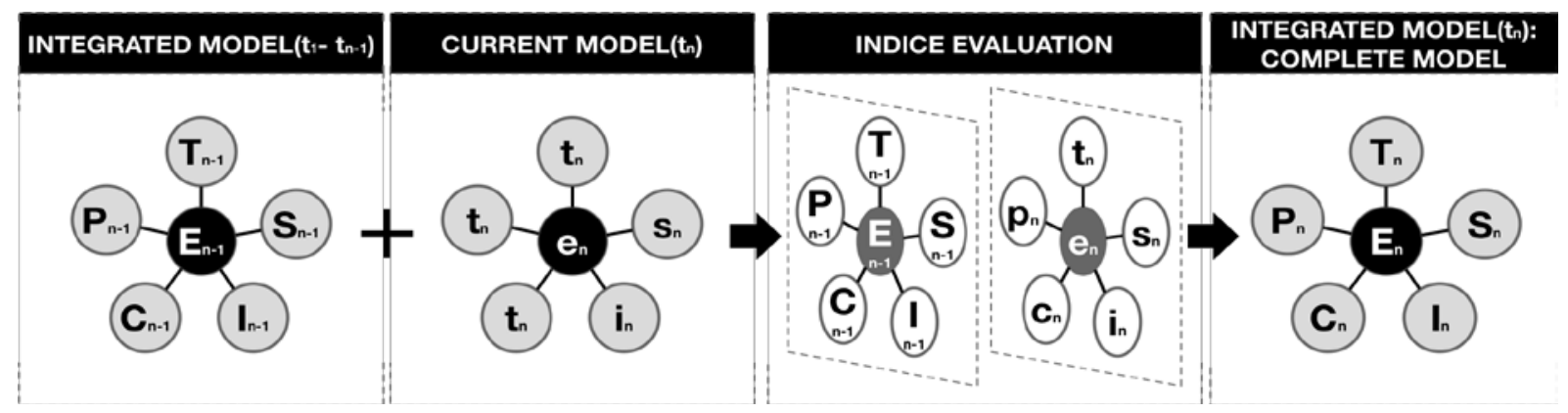

Figure 2: Event-indexing model (updating existing situation model with new event model

\subsection{New Information Exchange Environment with Social Media}

Before the emergence of Web 2.0, information is predominantly generated by mainstream media such as newspapers, radio, television and publishing. The public receive information in a passive manner and there were limited channels for individuals to express opinions or communicate with others. The interactive Web 2.0 based technologies have facilitated a multitude of digital media and technologies, which allow users to create and share content and to act collaboratively (Gruber, 2008; Schoder, Gloor, \& Metaxas, 2013), and therefore is called social media. Example social media platforms include micro-blogging sites such as Twitter and Sina Weibo, business- and employment-oriented social networking service such as Linkedin, social question and answer platforms such as Quora and Zhihu, photo-sharing applications such as Instagram and Tumblr, and video exchanging platforms such as Youtube.

The rapid growth of Social Media has immense impact on how people communicate and interact. Research indicate that with online social activities becoming ubiquitous, a significant and growing number of people receive and spread mainstream media data through social media (Hermida, Fletcher, Korell, \& Logan, 2012). Social media has changed the way people consume and diffuse information. Information is received though a set of highly personalized 
channels (Hermida et al., 2012), and is reshaped and interpreted in a more individual, segmental and real-time manner, and is spread at extremely high speed reaching a much wider range of receivers. Furthermore, the social network structure of data users also affects the way how information is spread, accumulated and processed. Therefore, the interpretation of mainstream media content is influenced by ongoing and dynamic contribution of opinions by involved users empowered by social media.

To understand how various types of social media services have reshaped the interactions among online users, we refer to the seven functionalities of social media illustrated by the honeycomb framework (Figure 3), including identity, presence, sharing, conversations, relationships, reputation and groups (Kietzmann et al., 2011). These functionalities explain the varieties of strategic engagement of social media services. For instance, LinkedIn users are thought to care mostly about identity, reputation, and relationships, whereas Twitter's primary features are sharing, conversations, groups and reputation.
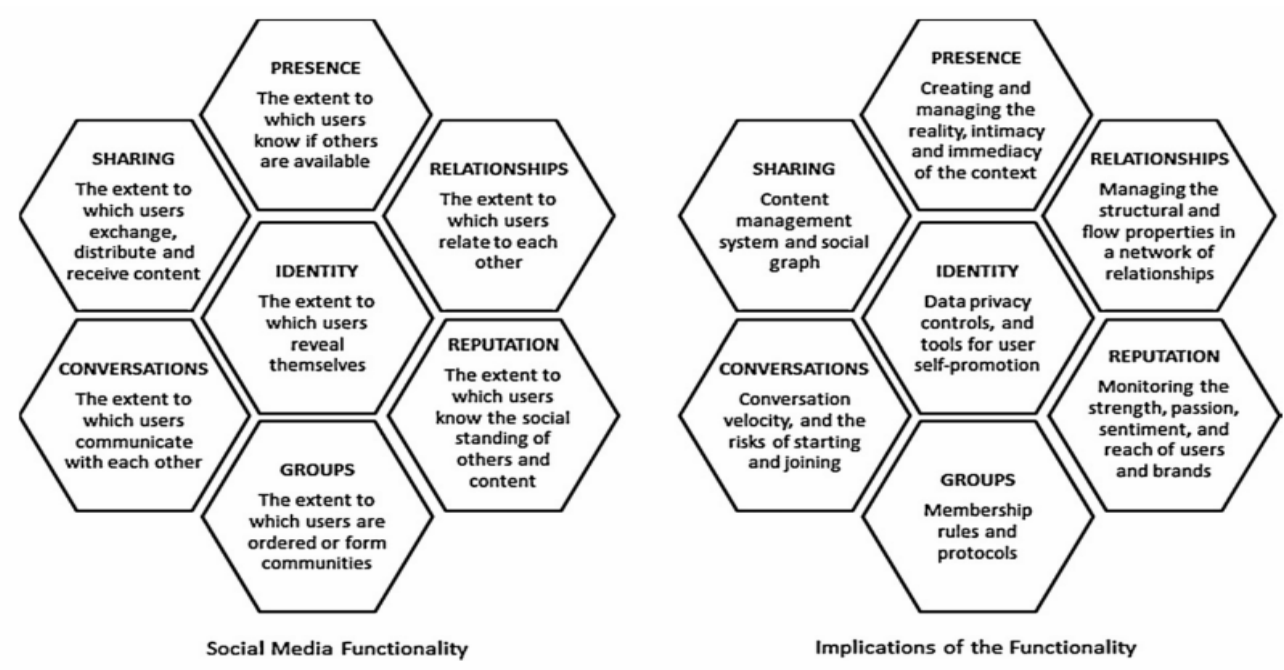

Figure 3: The honeycomb of social media (Kietzmann, Hermkens, McCarthy, E Silvestre, 2011)

The honeycomb framework was proposed to help business managers to understand the social media landscape so as to develop suitable strategies for their social media activities and content accordingly (Kietzmann et al., 2011). It also portrays social media as the enabler of another layer of the information ecology, as called social layer in this study, which provides value for investigating how information is consumed, generated and diffused in the global online communication environment. 'Identity and reputation' imply the influence of the users. 'Presence, relationships and groups' highlight the pattern of interactions of online communities. 'Sharing and conversations' focus on the dynamic and real-time features of the communications. This classification of the functionalities of social media indicates the significance of the roles users and the features of information transmissions. Firstly, social media content has become an increasingly important information source in addition to the traditional mainstream data such as newspapers and publications. Secondly, the interpretation of mainstream data is now influenced dynamically overtime due to the involvement of online communities, especially the influencer's opinions. Lastly, the social network structure and the influence factors of users have significant impact on the manner information is reprocessed and diffused. The accumulated information upon the connectivity of friends, subscriptions 
and groups now has significant impact and reshapes the conventions of data consumption (Figure 4).

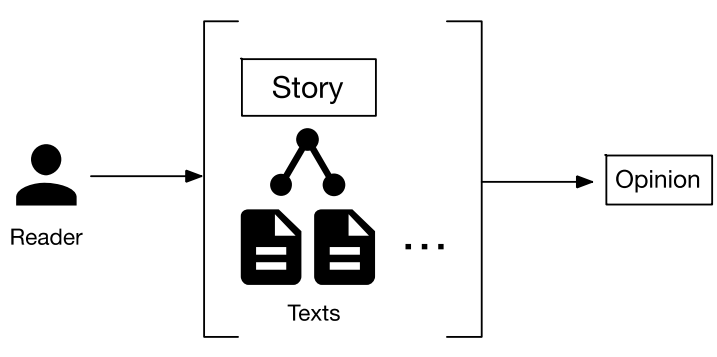

Traditional text processing environment

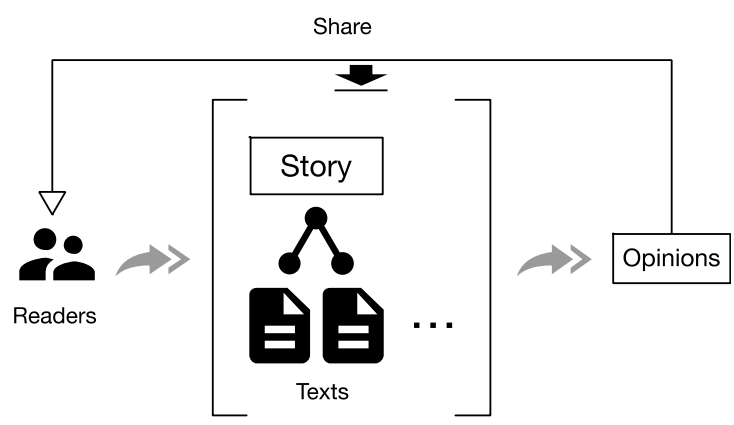

Text processing environment with social media

Figure 4: Comparison of traditional text processing and text processing with social media

\subsection{Rethinking of Situation Model Theory with Social Media}

Situation model theory was proposed for traditional discourse comprehension, especially narrative text understanding by the human mind (Walter Kintsch \& Van Dijk, 1978; Van Dijk, 1977; Rolf A Zwaan \& Gabriel A Radvansky, 1998). The strength of the situation models is to extract, integrate and model fragmental situational information from one or more texts into a coherent set of beliefs, which represents what the processed text(s) is about (Walter Kintsch \& Van Dijk, 1978; Rolf A Zwaan et al., 1995; Rolf A Zwaan \& Gabriel A Radvansky, 1998). Notably the successful construction of situation models relies heavily on prior acquired knowledge and experiences to perform inferring operations and fill up the gaps of literal expressions (Barclay, Bransford, Franks, McCarrell, \& Nitsch, 1974; Graesser, Gernsbacher, \& Goldman, 2003; Graesser et al., 1997; Walter Kintsch, 1988). Researchers claim that comprehension is always to some extent idiosyncratic because when readers elaborate the situational models they refer to the most appropriate and useful personal knowledge and experiences (Anderson, 1984; Langer, 1984; Tannen, 1981).

Although situation model explains how human mind decode meanings sentence by sentence and construct holistic and coherent mental representations about the text, or even across a set of texts, the original model does not cater to fuse scattered, segmented, heterogeneous and subjective event related information created by large numbers of users. Neither does it represent the dynamic augmentation of social views overtime. Therefore, based on the investigation of information exchange behaviours in social media, we have adapted the existing cognitive models of text comprehension to cater to the new environment of globalized online conversations.

To examine the influences of social media and how individuals and opinions are organized and interact dynamically, we examined the honeycomb framework of social web (Figure 3) which illustrate seven key functionalities of social media in assisting individual content creation and diffusion. Different social media platforms focus on various combinations of these functional areas depending on corresponding application requirements. The framework reveals the importance of both the structure of how people are connected and the role in the community (identity, relationships, presence, reputation, groups) and the way that users exchange comments about subject topics (sharing, conversations). Considering the honeycomb framework, it could be seen that social media differs from previously described 
narrative text. A key difference is that social media information is a 'conversation' which is one of the dimensions of the honeycomb framework. Referring to another dimension 'sharing', we could see that the information is shared and such sharing is impacted by a) the identity of writer, (b) the groups the writer belongs to, (c) the reputation of the writer, (d) presence on the social network and the (e) 'reputation' on the social network as well as in society. Each of these factors have been identified in the honeycomb framework as such we use the framework as a foundation to the claim that traditional narrative text develops into a conversation with dynamics dictated by the components of the honeycomb framework when considering social media information. The finding highlights the necessity of re-evaluating the dimensions of situation models. Under the new information environment with social web, the understanding of events is not only coordinated by the direct protagonist involved in the events, but also indirect online observers with diverse forms of actions, such as viewing, commenting, sharing. Although the indirect viewers are not necessary for situation model construction, their contribution has significant impact on the subject factors of events.

\subsubsection{Subjectivity and Objectivity of Situation Models}

Although situation model serves as the fundamental units for event construction, the five situational dimensions' functions differently and are extracted from various information sources. Temporality and spatiality in combination servers as the essential situational framework to distinguish events (Rolf A Zwaan, 1999; Rolf A Zwaan et al., 1995). Protagonist is an important clue that lead the thread of events to understand the plot of the story (Radvansky \& Zacks, 2014; Zacks, Speer, Swallow, Braver, \& Reynolds, 2007; Rolf A Zwaan, 1999). However, causality and intentionality are often not literally conveyed in the source text, which require the reader to interpreted with emotions, pre-acquired knowledge and experiences (Rolf A Zwaan \& Gabriel A Radvansky, 1998), which can be subjective and liable to change with new information (Van Dijk, 1982). The interpretation outcomes vary amongst individuals and are largely influenced by psychological status and social opinions. Van Dijk (1982) defined subjective understanding of the situation conveyed in a text as the beliefs, opinions or attitudes that are formed or activated with reference to the referents of the discourse (i.e. an event, a situation or participants). Therefore, we classify causality and intentionality as subjective dimensions, and the temporality, spatiality and protagonist as objective dimension, which is free of individual's influence (Figure 5).

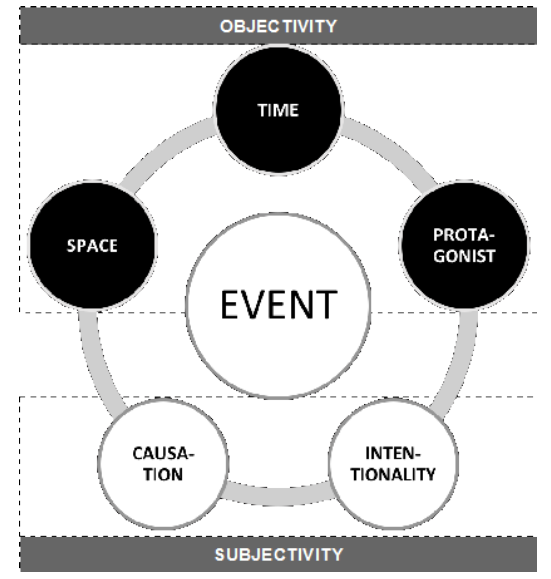

Figure 5: Subjectivity and objectivity of situation models 
Unlike objective dimensions which is always declarative and static in texts, subjective factors are not always directly presented in the source text and are constructed with background knowledge and experiences, psychological conditions and impact of social knowledge. Moreover, in an information ecosystem impacted and influenced by social media data, event related information being interpreted is no longer purely dependent on the original source text. In many cases, the subjective factors related to an event described in a source article is subject to re-evaluation and interpretation based on social viewpoints related to the event and individual experiences and knowledge overtime. Therefore, the collective and augmentation of the social view is subject to change overtime accordingly.

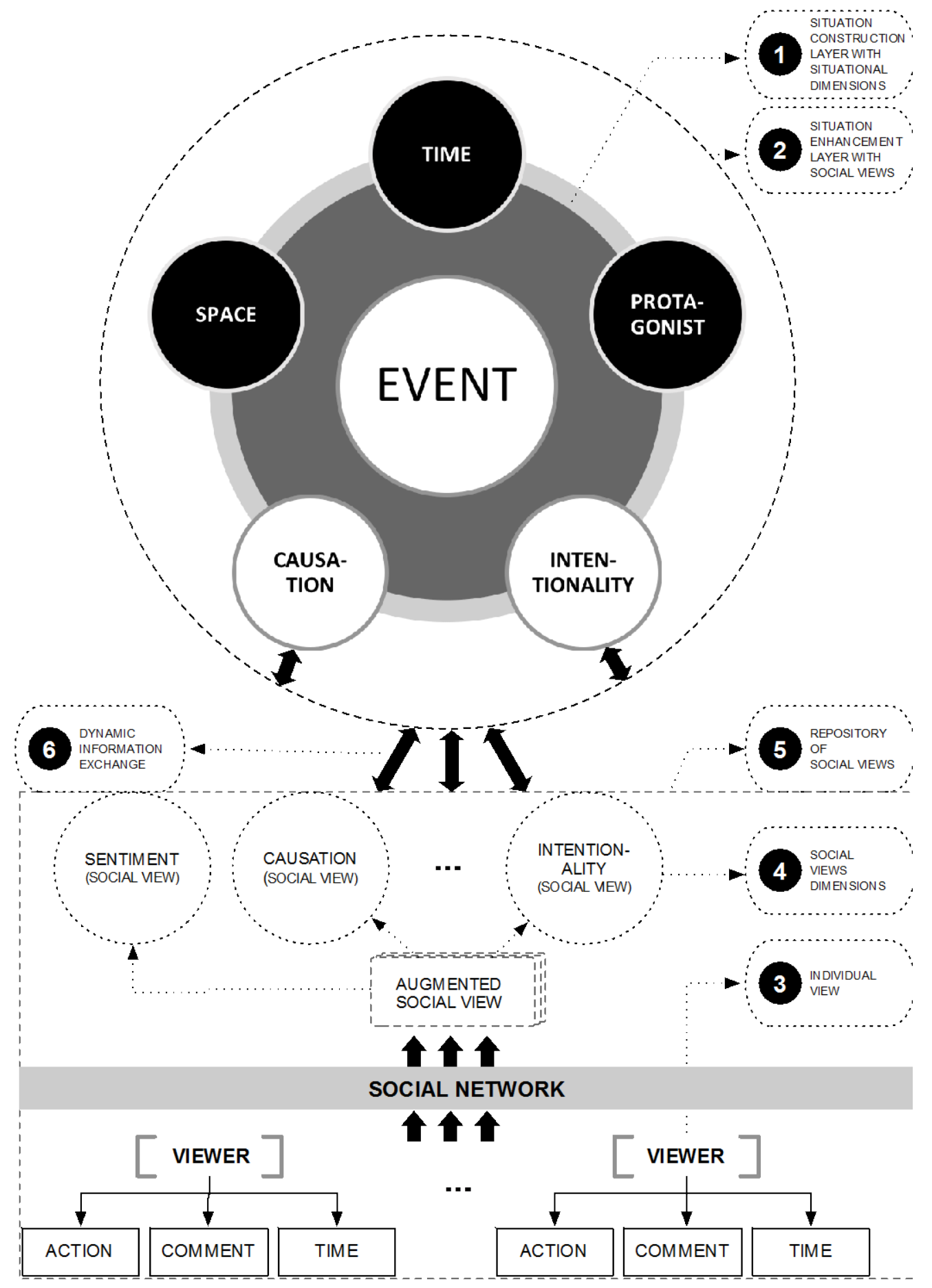

Figure 6: Extended situation model 
Extended situation models

To adapt the situation models into the global conversation environment and to model the knowledge ecosystem empowered by social media, we enhanced the existing model with the following features (Figure 6):

1. The original situation models layer serves as the core framework of events for knowledge construction and enhancement.

2. The social view layer represents the collective opinion of the society.

3. Individual opinions related to events are extracted from contents or activates generated by individual online users and anchored to corresponding events based on eventindexing theory.

4. The streams of individual opinions are analysed, categorized, and augmented as the collective social views, such sentiment, causation, intentionality to support analysis at more granular level.

5. The source comment, extracted individual opinions, categorized and augmented social opinions serve as the social opinion repository.

6. The collective social views are coordinated with related situation model of events, which provides an enriched layer of understanding of the subjective dimensions of the events. On the other hand, the evolution of social opinion also influence the outcome of individual interpretation of an event.

\section{Construction of the Adapted Situation Models}

\subsection{Sample Data Collection}

To demonstrate and validate the extended situation model, we gathered a sample dataset from Twitter published by New York Times' official accounts (nytimes) about the topic "Turkey coup" during 2016-07-15 to 2017-07-16. 33 tweets were collected, analysed and applied to the proposed model (Appendix 1).

\subsection{Core Situation Models Construction}

The core situation models construction follows the multi-layer processes from surface code, textbase to situation models.

Firstly, the explicit wording and syntactical rules were analysed to identify individuals, objects, concepts and the corresponding relations. Then propositions are extracted based on the surface layer analysis to be networked as textbase via overlapping dimensions as illustrated in Figure 7. 
Sample tweet: Erdogan blamed the coup attempt on Fethullah Gulen and his followers. Gulen lives in exile in Pennsylvania. (17/07/16)

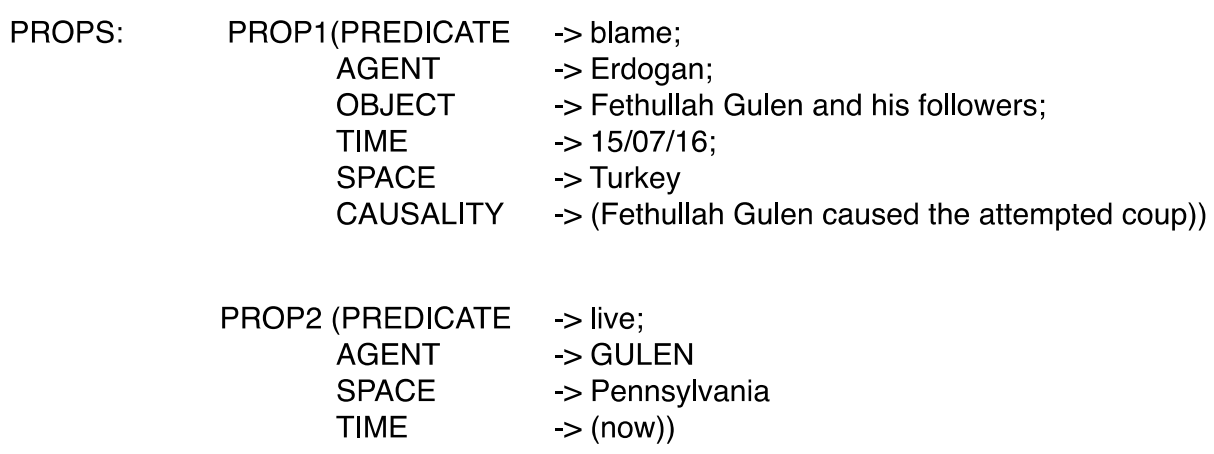

Figure 7: Textbase extraction example

The situational dimensions including time, space, protagonist (people, organization), causality and intentionality were extracted based on the textbase, so that event and event-related dimensions are integrated and coordinated according to similarities of situational settings along the orderable tempo-spatial frameworks.

In this experiment, significant situational dimensions were identified based on the frequencies of occurrence in the texts and plotted in a networked representation of situation models. Two tempo-spatio situation frameworks are embedded in the investigated sample text:[TIME=15/07/16; SPACE=TURKEY] and [TIME=16/07/16; SPACE=TURKEY]. The top frequent protagonists include Recep Tayyip Erdogan, Fethullah Gulen, the military and Binali Yildirm.

Given situation framework [TIME=15/07/16; SPACE=TURKEY], the related events are connected via predicates or verbs as the central building blocks which links the propositional information and establishes coherence of understanding (Figure 8). There are two subjective factors revealed in the illustrated model, as shown in the left section of Figure 8. Noticeably the causality factor [C: Fethullah Gulen has a causal role in the coup] demonstrates contradictory views from two tweets (Table 1). 


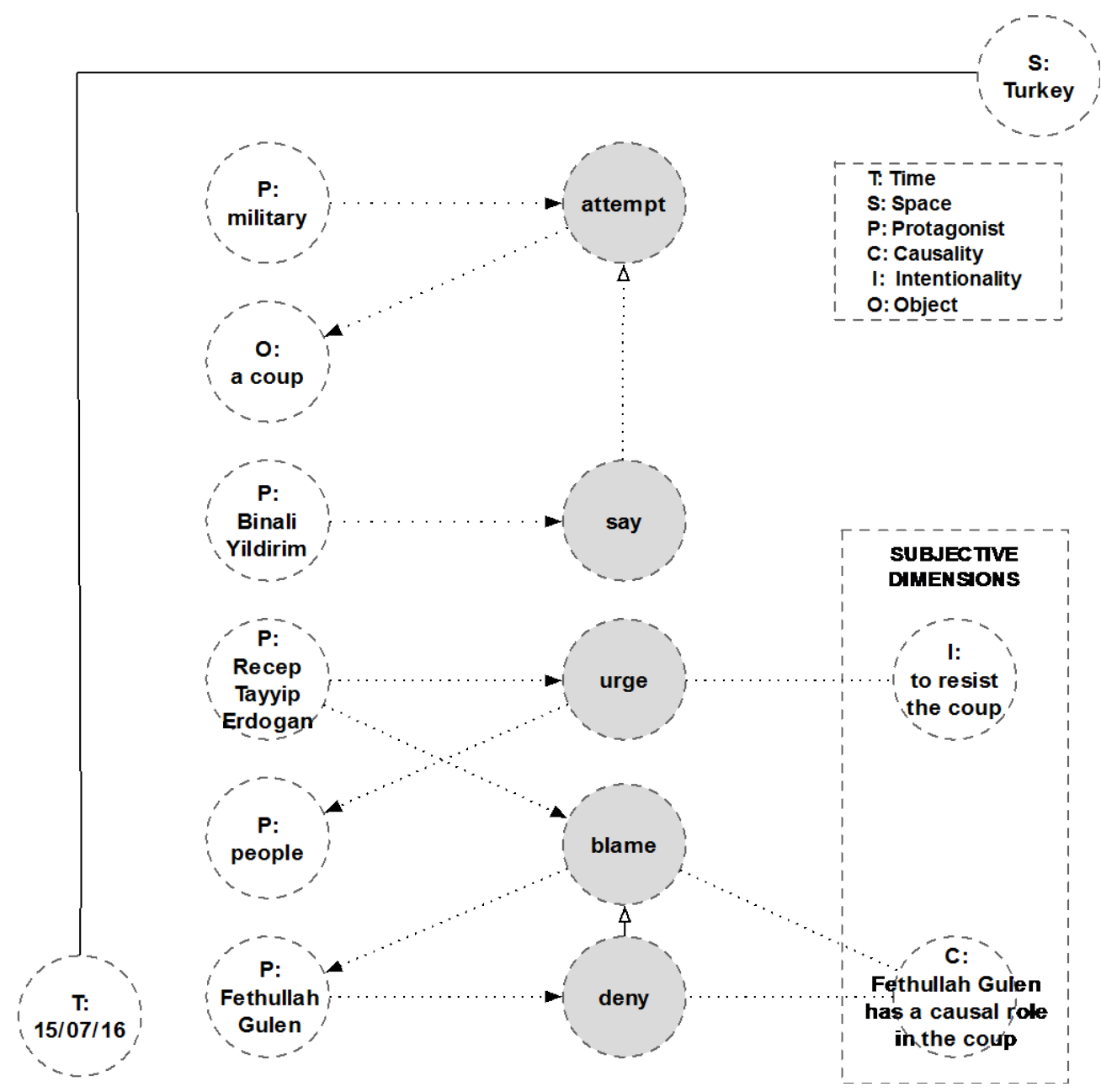

Figure 8: Integrated Situation model [TIME=15/07/16; SPACE=Turkey]

\begin{tabular}{l|l}
\hline Tweet ID: 8 & $\begin{array}{l}\text { Erdogan blamed the coup attempt on Fethullah Gulen and his } \\
\text { followers. Gulen lives in exile in Pennsylvania. }\end{array}$ \\
Tweet ID: 17 & $\begin{array}{l}\text { Fethullah Gulen, an exiled Erdogan opponent, denies role in Turkey } \\
\text { coup attempt }\end{array}$
\end{tabular}

Table 1: Example tweets being examined for the subjective factor

Without external knowledge, the integrated situation model itself is insufficient to provide a clear view about the subjective factor. Therefore, we introduce additional crowd opinions about the event to enhance the existing knowledge to clarify the ambiguous dimension with the adaptive situation models.

\subsection{Adaptive situation model extension}

To obtain more knowledge about the subjective causality dimension of "Fethullah Gulen has a causal role in the coup", we extracted users' 46 comments in English about two tweets (Table 1) related to the stated causality of the events (Appendix 2).

Comments were categorized as Positive, Negative or Neutral opinions depending on relevance with the investigated subjective factor (Appendix 2). For each comment $\left(c_{i}\right)$ in given comment set $C$, if the opinion support subjective factor in Positive, Negative or Neutral 
manner, we assign value 1 under corresponding category, and the rest categories with value 0 . We define number of 'likes' for comment $c_{i}$ as $l_{i}$. and the augmented Positive, Negative and Neutral opinions as $O_{\text {pos, }} O_{\text {neg }}$ and $O_{\text {neu: }}$ :

$$
\begin{aligned}
& o_{\text {pos }}=\sum_{i \in C}\left(\text { pos }_{i} \times l_{i}\right) \\
& O_{\text {neg }}=\sum_{i \in C}\left(\text { neg }_{i} \times l_{i}\right) \\
& O_{\text {neu }}=\sum_{i \in C}\left(\text { neu }_{i} \times l_{i}\right)
\end{aligned}
$$

We define confidence level of the augmented Positive, Negative and Neutral opinion as:

$$
\begin{aligned}
& \text { Con }_{\text {pos }}=\frac{o_{\text {pos }}}{\sum_{i \in C}\left(l_{i}+1\right)} \\
& \text { Con }_{\text {neg }}=\frac{o_{\text {neg }}}{\sum_{i \in C}\left(l_{i}+1\right)} \\
& \text { Con }_{\text {neu }}=\frac{o_{\text {neu }}}{\sum_{i \in C}\left(l_{i}+1\right)}
\end{aligned}
$$

The augmented confidence level for Positive, Negative or Neural view about "Fethulah Gulen's causal role" are $0.41,0.21$ and 0.38 respectively. The results indicate that $41 \%$ users hold a positive view of 'Fethulah Gulen's causal role' in the Turkey coup, and 21\% and 38\% hold Negative and Neural view respectively. It implies that although the official tweets from New York Times present contradictory information and ambiguous judgement about the examined subjective factor, the involved social media community demonstrate strong bias that positively supports the view.

\section{Discussion}

This paper has (a) proposed a novel the representation of event-centric knowledge by extending situational and event extending models from psychology (b) outlined a mechanism of how events can be constructed and updated with continuous textual information streamed from heterogeneous forms of media especially magnified by social media, and (c) emphasised how the mechanism could result in enhancing the understanding of the subjective aspects of events with the dynamics of social opinions. We re-evaluated the definitions of events and borrowed ideas from psychological researches in text comprehension and event cognition fields. This study is based on the event-indexing situation model theory which construct events with five situational dimensions and utilize them as indices for event inference and updating. Acknowledging the strength of situation model for capturing situational context to coordinate the integration of segmental text into coherent set of beliefs, we distinguished the subjective and objective natures of the situational dimensions and the different types of knowledge and the sources of information for such knowledge these dimensions are built upon. We also reiterate the importance of enhancing the understanding of subjective factors of events with social knowledge. Therefore, we have extended the model with a social view layer to represent the interactive and evolutional features of social opinions from social media.

The purpose of the adapted situation model is to 1) capture events with situational context, 2) establish associations of events with involved elements and associations across events, 3) revealing evolution of events with time features, 4) coordinate dynamic social opinions to corresponding events through situational dimensions and enrich the necessary social knowledge with the augmented social opinions over time, 5) enable fluid knowledge 
augmentation through online construction and updating of events with textual sources of diverse formats streaming from heterogeneous media.

We have experimented the model with a sample of real data gathered from New York Times's official twitter account. The case study demonstrated the construction of situation models via layered text analysis and representations. With situation model, we have re-established the events with corresponding situational settings back into sequential orders, i.e. temporal or causal order. It also reflected the contradictory views of subjective factors among different parties involved. Relevant social opinions were extracted and analysed, and was anchored to the specific subjective dimensions of the related events to enrich the social knowledge aspect. We observed that the social opinion layer of the model indicates a noticeable biased view of the examined subjective factor, which could be quite dissimilar from the views conveyed by the investigated mainstream media. The extended social knowledge provides a means to investigate the influencing and recursive interactions between the core situation model layer and the social view layer. We did not elaborate the model with this feature as it was out of scope for the research presented in the paper.

The dynamics of individual social view plays an increasingly important role in the evolution of public opinion as a collective impact factor. Researches also demonstrate that emotions embedded in the contents (Berger \& Milkman, 2012), types and size of media, interaction patterns of the information system are all contributive factors on the formation, trends and diffusion of the public opinions(Quattrociocchi, Caldarelli, \& reports, 2014). How to recognize the behavioural patterns of information exchanging between the situation model layer and social view layer and how public and individual opinions evolves accordingly is still an open question and attracts continuous research interests in this area (Bakshy, Messing, \& Science, 2015; Berger \& Milkman, 2012; Quattrociocchi et al., 2014).

\section{References}

Adolphs, R. (2009). The social brain: neural basis of social knowledge. Annual review of psychology, 60, 693-716.

Aguilar, J., Beller, C., McNamee, P., Van Durme, B., Strassel, S., Song, Z., \& Ellis, J. (2014). A comparison of the events and relations across ace, ere, tac-kbp, and framenet annotation standards. Paper presented at the Proceedings of the Second Workshop on EVENTS: Definition, Detection, Coreference, and Representation.

Ahn, D. (2006). The stages of event extraction. Paper presented at the Proceedings of the Workshop on Annotating and Reasoning about Time and Events.

Allan, J. (2002). Introduction to topic detection and tracking. Topic detection and tracking, 1-16.

Allan, J., Carbonell, J. G., Doddington, G., \& Yamron, J. (1998). Topic detection and tracking pilot study final report. Topic detection and tracking pilot study final report.

Anderson, R. C. (1984). Role of the reader's schema in comprehension, learning, and memory. Learning to read in American schools: Basal readers and content texts, 29, 243-257.

Baker, C. F., Fillmore, C. J., \& Lowe, J. B. (1998). The berkeley framenet project. Paper presented at the Proceedings of the 36th Annual Meeting of the Association for Computational Linguistics and 17th International Conference on Computational Linguistics-Volume 1. 
Bakshy, E., Messing, S., \& Science, A.-L. A. (2015). Exposure to ideologically diverse news and opinion on Facebook. Science.

Barclay, J. R., Bransford, J. D., Franks, J. J., McCarrell, N. S., \& Nitsch, K. (1974). Comprehension and semantic flexibility. Journal of Verbal Learning and Verbal Behavior, 13(4), 471-481.

Berger, J., \& Milkman, K. L. (2012). What makes online content viral? Journal of marketing research, 49(2), 192-205.

Brüggemann, D., Hermey, Y., Orth, C., \& Schneider, D. (2016). Topic Detection and Tracking System. Topic Detection and Tracking System.

Cambria, E., \& White, B. (2014). Jumping NLP curves: A review of natural language processing research. IEEE Computational intelligence magazine, 9(2), 48-57.

Chambers, N., \& Jurafsky, D. (2008). Unsupervised Learning of Narrative Event Chains. ACL.

Crowley, J. L., Reignier, P., \& Barranquand, R. (2009). Situation models: A tool for observing and understanding activity. Paper presented at the in Workshop People Detection and Tracking, held in IEEE International Conference on Robotics and Automation, Kobe, Japan.

Dijk, V. T. A. (1977). Semantic macro-structures and knowledge frames in discourse comprehension. Cognitive processes in comprehension.

Dijk, V. T. A., Kintsch, W., \& Dijk, V. T. A. (1983). Strategies of discourse comprehension. Strategies of discourse comprehension.

Doddington, G. R., Mitchell, A., Przybocki, M. A., Ramshaw, L. A., Strassel, S., \& Weischedel, R. M. (2004). The Automatic Content Extraction (ACE) Program-Tasks, Data, and Evaluation. Paper presented at the LREC.

Gangemi, A., Hassan, E., Presutti, V., \& Reforgiato, D. (2013). Fred as an event extraction tool. Paper presented at the Proceedings of the Workshop on Detection, Representation, and Exploitation of Events in the Semantic Web.

Garnham, A. (1987). Mental models as representations of discourse and text: Ellis Horwood.

Graesser, A. C., Gernsbacher, M. A., \& Goldman, S. R. (2003). Handbook of discourse processes: Routledge.

Graesser, A. C., Millis, K. K., \& Zwaan, R. A. (1997). Discourse comprehension. Annual review of psychology, 48(1), 163-189.

Gruber, T. (2008). Collective knowledge systems: Where the social web meets the semantic web. Web semantics: science.

Hermida, A., Fletcher, F., Korell, D., \& Logan, D. (2012). Share, like, recommend: Decoding the social media news consumer. Journalism Studies, 13(5-6), 815-824.

Hogenboom, F., Frasincar, F., Kaymak, U., De Jong, F., \& Caron, E. (2016). A survey of event extraction methods from text for decision support systems. Decision Support Systems, 85, 12-22.

Johnson-Laird, P. N. (1983). Mental models: Towards a cognitive science of language, inference, and consciousness: Harvard University Press. 
Kietzmann, J. H., Hermkens, K., McCarthy, I. P., \& Silvestre, B. S. (2011). Social media? Get serious! Understanding the functional building blocks of social media. Business horizons, 54(3), 241-251.

Kingsbury, P., \& Palmer, M. (2002). From TreeBank to PropBank. Paper presented the LREC.

Kintsch, W. (1988). The role of knowledge in discourse comprehension: A constructionintegration model. Psychological review, 95(2), 163.

Kintsch, W. (1998). Comprehension: A paradigm for cognition: Cambridge University Press.

Kintsch, W., \& Dijk, V. T. A. (1978). Toward a model of text comprehension and production. Psychological review.

Kintsch, W., \& Van Dijk, T. A. (1978). Toward a model of text comprehension and production. Psychological review, 85(5), 363.

Langer, J. A. (1984). Examining background knowledge and text comprehension. Reading Research Quarterly, 468-481.

Piaget, J., \& Duckworth, E. (1970). Genetic epistemology. American Behavioral Scientist, 13(3), 459-480.

Quattrociocchi, W., Caldarelli, G., \& reports, S.-A. (2014). Opinion dynamics on interacting networks: media competition and social influence. Scientific reports. doi:10.1038/srep04938

Radvansky, G. A., \& Zacks, J. M. (2014). Event cognition: Oxford University Press.

Sanford, A. J., \& Emmott, C. (2012). Mind, brain and narrative: Cambridge University Press.

Schoder, D., Gloor, P. A., \& Metaxas, P. T. (2013). Social media and collective intelligenceongoing and future research streams. KI-Künstliche Intelligenz.

Tanev, H., Piskorski, J., \& Atkinson, M. (2008). Real-time news event extraction for global crisis monitoring. Natural Language and Information Systems, 207-218.

Tannen, D. (1981). Indirectness in discourse: Ethnicity as conversational style. Discourse processes, 4(3), 221-238.

Van Dijk, T. A. (1977). Semantic macro-structures and knowledge frames in discourse comprehension. Cognitive processes in comprehension, 332.

Van Dijk, T. A. (1982). Opinions and attitudes in discourse comprehension. In Advances in Psychology (Vol. 9, pp. 35-51): Elsevier.

Zacks, J. M., Speer, N. K., Swallow, K. M., Braver, T. S., \& Reynolds, J. R. (2007). Event perception: a mind-brain perspective. Psychological bulletin, 133(2), 273.

Zwaan, R. A. (1999). Five dimensions of narrative comprehension: The event-indexing model. Narrative comprehension, causality, and coherence: Essays in honor of Tom Trabasso, 93-110.

Zwaan, R. A. (2016). Situation models, mental simulations, and abstract concepts in discourse comprehension. Psychonomic bulletin \& review.

Zwaan, R. A., Langston, M. C., \& Graesser, A. C. (1995). The construction of situation models in narrative comprehension: An event-indexing model. Psychological science. doi:10.2307/40063035 
Zwaan, R. A., Langston, M. C., \& Graesser, A. C. (1995). The construction of situation models in narrative comprehension: An event-indexing model. Psychological science, 6(5), 292297.

Zwaan, R. A., \& Radvansky, G. A. (1998). Situation models in language comprehension and memory. Psychological bulletin, 123(2), 162-185. doi:10.1037/0033-2909.123.2.162

\section{Appendices}

\section{Appendix 1: Sample tweets of topic "Turkey coup" (15/07/16-16/07/16))}

\begin{tabular}{|c|c|c|}
\hline ID & Publishi Date & Text \\
\hline 1 & $15 / 7 / 16$ & Breaking News: Turkey's prime minister said that factions of the military had attempted a coup \\
\hline 2 & $15 / 7 / 16$ & Turkey's prime minister said a military faction was attempting a coup http://nyti.ms/29CAYRJ \\
\hline 3 & $15 / 7 / 16$ & A look at Erdogan's controversial rule in Turkey, published just days ago \\
\hline 4 & $15 / 7 / 16$ & Erdogan: "There is no power higher than the power of the people." \\
\hline 5 & $15 / 7 / 16$ & "There's a coup," one man shouted in the street. "There's a coup, and blood will be shed." \\
\hline 6 & $15 / 7 / 16$ & Breaking News: Turkey's president used an iPhone to urge people to resist a coup attempt \\
\hline 7 & $15 / 7 / 16$ & Twitter: "We suspect there is an intentional slowing of our traffic" in Turkey \\
\hline 8 & $15 / 7 / 16$ & Erdogan blamed the coup attempt on Fethullah Gulen and his followers. Gulen lives in exile in Pennsylvania. \\
\hline 9 & $15 / 7 / 16$ & Tumult in Turkey: What we know and what we don't know \\
\hline 10 & $15 / 7 / 16$ & Who is Fethullah Gulen, and what is his role in the coup attempt in Turkey? \\
\hline 11 & $15 / 7 / 16$ & Turkey's state-run news agency: 17 police officers killed in helicopter attack on police special forces headquarters \\
\hline 12 & $15 / 7 / 16$ & Follow the latest updates on the attempted coup in Turkey \\
\hline 13 & $15 / 7 / 16$ & President Erdogan of Turkey has returned to Istanbul, signaling that the military coup is faltering \\
\hline 14 & $15 / 7 / 16$ & 12 killed in an explosion at the Turkish Parliament building, CNN Turk reports \\
\hline 15 & $15 / 7 / 16$ & Soldiers in Turkey reportedly take over newspaper; CNN studio is briefly seized \\
\hline 16 & $15 / 7 / 16$ & At least 60 dead in Turkey, and 754 military members reported arrested \\
\hline 17 & $15 / 7 / 16$ & Fethullah Gulen, an exiled Erdogan opponent, denies role in Turkey coup attempt \\
\hline 18 & $16 / 7 / 16$ & 90 people have been killed in Turkey overnight, with more than 1,100 wounded. More than 750 have been arrested. \\
\hline 19 & $16 / 7 / 16$ & As President Erdogan confronts mutiny in Turkey, U.S. finds itself on shakier ground \\
\hline 20 & $16 / 7 / 16$ & Photos show clashes on the Bosporus Bridge in Istanbul \\
\hline 21 & $16 / 7 / 16$ & Soldiers tried to pile into a bus to escape a mob on the Bosporus Bridge in Istanbul, Turkey \\
\hline 22 & $16 / 7 / 16$ & Tumult in Turkey: What we know and don't know \\
\hline 23 & $16 / 7 / 16$ & Death toll in Turkey rises to at least 265 . Security forces detain 2,639 military personnel. \\
\hline 24 & $16 / 7 / 16$ & "I'm very afraid because in the past a lot of innocent blood was shed in these coups," a teacher in Istanbul said \\
\hline 25 & $16 / 7 / 16$ & Turkey's government detains thousands of military personnel in a bid to regain control after a night of chaos \\
\hline 26 & $16 / 7 / 16$ & What we know and don't know about the attempted coup in Turkey \\
\hline 27 & $16 / 7 / 16$ & Kerry, on Turkish coup attempt: "I must say it does not appear to be a very brilliantly planned or executed event" \\
\hline 28 & $16 / 7 / 16$ & Turkish officials halted American-led strikes against the Islamic State from a major air base \\
\hline 29 & $16 / 7 / 16$ & Turkish coup attempt: - Death toll at 265 - Thousands in military detained - U.S.-led strikes against ISIS halted \\
\hline 30 & $16 / 7 / 16$ & The morning after a failed coup in Turkey \\
\hline 31 & $16 / 7 / 16$ & Fetullah Gulen gave an interview to media at his Saylorsburg, Pa compound. NYT's Stephanie Saul filmed it: \\
\hline 32 & $16 / 7 / 16$ & What we know and don't know about the attempted coup in Turkey \\
\hline 33 & $16 / 7 / 16$ & Turkey woke up to the aftermath of a coup attempt that left more than 250 people dead \\
\hline
\end{tabular}




\section{Appendix 2: Examine subjective dimension C(ausality) with example}

\begin{tabular}{|c|c|c|c|c|c|c|}
\hline \multicolumn{7}{|c|}{ Subjective View: Fethullah Gulen has a causal role in the coup } \\
\hline $\begin{array}{l}\vec{\S} \\
\mathbb{\$} \\
\stackrel{\mathbb{P}}{+} \\
\bar{\sigma}\end{array}$ & 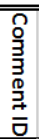 & Comments & 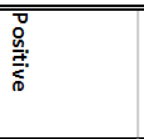 & 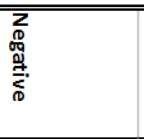 & 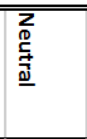 & 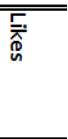 \\
\hline \multirow[t]{27}{*}{8} & 1 & will US admin hand over this criminal to turkey if it is proved his role in this coup? I doubt & 1 & 0 & 0 & 4 \\
\hline & 2 & do you really believe a dictator who banned free press, protests, and YouTube? & 0 & 1 & 0 & 1 \\
\hline & 3 & Bo s friend & 0 & 0 & 1 & 0 \\
\hline & 4 & $\begin{array}{l}\text { Let's hope now there won't be attacks in Pennsylvania since the NYTimes thinks it was } \\
\text { necessary to say where Gulen lives! }\end{array}$ & 0 & 0 & 1 & 3 \\
\hline & 5 & Who da thunk that a coup was being orchestrated from the Poconos! & 0 & 1 & 0 & 2 \\
\hline & 6 & and he runs charter school \$\$ scheme in US- big connections to Hillary & 0 & 0 & 1 & 1 \\
\hline & 7 & so he carried the coup from the USA, that's really amazing... & 0 & 1 & 0 & 1 \\
\hline & 8 & Connections in high places (?) & 0 & 0 & 1 & 0 \\
\hline & 9 & Right. When you're wrong, blame someone else? & 0 & 1 & 0 & 0 \\
\hline & 10 & Sounds familiar?: HRC \& BO & 0 & 0 & 1 & 1 \\
\hline & 11 & if \#erdogan survive the coup \#Turkey and US relation will be in trouble b/c of Gulen & 1 & 0 & 0 & 1 \\
\hline & 12 & @nytimes @morgfair is there *anything* Pennsylvania can't screw up? & 0 & 0 & 1 & 0 \\
\hline & 13 & $\begin{array}{l}\text { So, de-democratization will continue or even increase in \#Turkey. So sad! And many even } \\
\text { don't understand that. \#TurkeyCoup }\end{array}$ & 0 & 0 & 1 & 0 \\
\hline & 14 & $\begin{array}{l}\text { Gulen and his followers promote democracy and peace more than 40years. What is the } \\
\text { benefit of Gulenists from such a coup attempt? }\end{array}$ & 0 & 1 & 0 & 0 \\
\hline & 15 & $\begin{array}{l}\text { Gulen needs to be rooted OUT of PA. He doesn't live American ideals and wants to } \\
\text { destabliize the American culture and system. }\end{array}$ & 1 & 0 & 0 & 0 \\
\hline & 16 & don't trust any of them & 0 & 0 & 1 & 0 \\
\hline & 17 & nice to read tha POTUS is for Democracy. Executive orders, anyone?? & 0 & 0 & 1 & 0 \\
\hline & 18 & so Erdogan can keep his power with presidential system & 0 & 1 & 0 & 0 \\
\hline & 19 & $\begin{array}{l}\text { Erdogan tricks people in Turkey, he planned this game to change prime minister system to } \\
\text { presidential system!!! }\end{array}$ & 0 & 1 & 0 & 0 \\
\hline & 20 & Clintons!!! & 0 & 0 & 1 & 0 \\
\hline & 21 & Video taken in Bakırköy, Istanbul showing people in support of Erdogen. & 1 & 0 & 0 & 0 \\
\hline & 22 & ah yes blame foreign conspirators, classic & 0 & 1 & & 0 \\
\hline & 23 & Erdogan in Istanbul Ataturk Airport People stans behind Turkish President. & 0 & 0 & 1 & 0 \\
\hline & 24 & $\begin{array}{l}\text { If Turkish President is correct US based cleric in all probability is an Islamist, like ISIS, with } \\
\text { some support in Turkey \&its army }\end{array}$ & 0 & 0 & 1 & 0 \\
\hline & 25 & Must be pals with Soros? & 0 & 0 & 1 & 1 \\
\hline & 26 & Are the charter schools safe? & 0 & 0 & 1 & 0 \\
\hline & 27 & Gluten is not good for you! & 1 & 0 & 0 & 0 \\
\hline \multirow[t]{20}{*}{17} & 28 & USA fuels Terrorism by giving refuge to radicals & 1 & 0 & 0 & 0 \\
\hline & 29 & he will pay for this & 1 & 0 & 0 & 1 \\
\hline & 30 & $\begin{array}{l}\text { from his dirty mind,we can say that he is the organizer of this attempt against Erdogan. } \\
\text { all Euro countries have role in Turkey. }\end{array}$ & 1 & 0 & 0 & 1 \\
\hline & 31 & Indeed, I believe he was on Media asking 4 the coup 2B stopped. & 1 & 0 & 0 & 0 \\
\hline & 32 & $\begin{array}{l}\text { With zero credibility, when his pupils attempted to intern the president and did actually } \\
\text { bomb the parliament. }\end{array}$ & 1 & 0 & 0 & 0 \\
\hline & 33 & Easy enough to prove, if needs must. & 1 & 0 & 0 & 0 \\
\hline & 34 & SEND HIM BACK...We will love him & 1 & 0 & 0 & 0 \\
\hline & 35 & what had happened? & & 0 & 1 & 0 \\
\hline & 36 & We know the truth very well. Be honest! & 1 & 0 & 0 & 0 \\
\hline & 37 & $\begin{array}{l}\text { Erdogan has prevailed. That's terrible. Why do the Islamists always win and we always } \\
\text { lose? Obama chose the wrong side ...again... }\end{array}$ & 1 & 0 & 0 & 0 \\
\hline & 38 & $\begin{array}{l}\text { "Who, me? You must have me confused with somebody else." Those arrested will provide } \\
\text { names, true or not, to stop the torture. }\end{array}$ & 1 & 0 & 0 & 0 \\
\hline & 39 & NO NEED TO FIND ESCAPE GOAT & 0 & 1 & 0 & 0 \\
\hline & 40 & $\begin{array}{l}\text { "Our politics shouldnt concern u, ur not American! Hold a sec gotta tweet abt the shit } \\
\text { happening in Turkey, Iraq, Britain \& France" }\end{array}$ & 0 & 0 & 1 & 0 \\
\hline & 41 & traitor FETULLAH GULEN & 1 & 0 & 0 & 0 \\
\hline & 42 & $\begin{array}{l}\text { Hypocrisy:the common A'can Twitter practice of telling nonAmericans to stay out of US } \\
\text { politics, but NEVER taking their own advice. }\end{array}$ & 0 & 0 & 1 & 0 \\
\hline & 43 & He is a schizophrenic mentally ill and very useful for some people.. & 1 & 0 & 0 & 0 \\
\hline & 44 & Did he deny before or after it failed? & 0 & 0 & 1 & 0 \\
\hline & 45 & of course he denies, because the coup gone wrong... & 1 & 0 & 0 & 0 \\
\hline & 46 & YES,... AND OBAMA IS WHITE.. FETULLAH GULEN THE PIG IS DE SOURCE.... & 1 & 0 & 0 & 0 \\
\hline & & (2: & 0.41 & 0.21 & 0.38 & \\
\hline
\end{tabular}

Copyright: (C) 2018 Wang, Alahakoon \& De Silva. This is an open-access article distributed under the terms of the Creative Commons Attribution-NonCommercial 3.0 Australia License, which permits non-commercial use, distribution, and reproduction in any medium, provided the original author and AJIS are credited. 


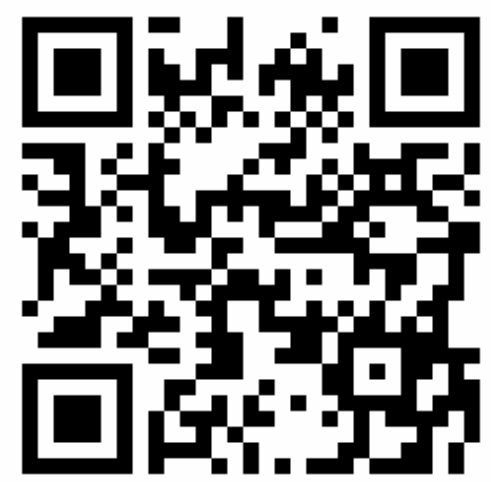

